\title{
MEROMORPHIC SOLUTIONS OF SOME FUNCTIONAL EQUATIONS
}

\author{
Walter Bergweiler, Katsuya Ishizaki, and Niro Yanagihara
}

\begin{abstract}
It is shown that transcendental meromorphic solutions $f(z)$ of the functional equation $\sum_{j=0}^{n} a_{j}(z) f\left(c^{j} z\right)=Q(z)$ where $0<|c|<1$ is a complex number and $a_{j}(z), j=0,1, \ldots, n$, and $Q(z)$ are rational functions with $a_{0}(z) \not \equiv 0$, $a_{n}(z) \equiv 1$, satisfy $T(r, f)=O\left((\log r)^{2}\right)$ and $(\log r)^{2}=O(T(r, f))$. Moreover, in the case $n=2$ and $Q(z) \equiv 0$, necessary and sufficient conditions for the existence of solutions are given.
\end{abstract}

\section{Introduction}

The functional equations of Schröder, Böttcher, and Abel have intimate relations to iteration theory and have been studied in detail in this context. In this note, we treat the functional equation

$$
\sum_{j=0}^{n} a_{j}(z) f\left(c^{j} z\right)=Q(z)
$$

where $0<|c|<1$ is a complex number and $a_{j}(z), j=0,1, \ldots, n$, and $Q(z)$ are rational functions with $a_{0}(z) \not \equiv 0, a_{n}(z) \equiv 1$. The equation has some similarity to the Schröder equation, but its study shows somewhat different aspects. We focus on the existence and the growth of solutions.

Throughout this paper, we use the value distribution theory. We use standard notations in the Nevanlinna theory (see, e.g., $[3,6,7])$. Let $f(z)$ be a meromorphic function. Here, and in the following, the word "meromorphic" means meromorphic in $|z|<\infty$. Let $M(r, f), m(r, f), n(r, f), N(r, f)$, and $T(r, f)$ denote the maximum modulus, the proximity function, the unintegrated counting function, the counting function, and the characteristic function of $f(z)$, respectively.

In this section, we state two results of the growth of meromorphic solutions of (1.1), which are proved in Section 2. In Section 3, we are concerned with an existence theorem of meromorphic solutions for the case $n=2$ and $Q(z) \equiv 0$ in (1.1), i.e.,

$$
f\left(c^{2} z\right)+a(z) f(c z)+b(z) f(z)=0
$$

where $a(z), b(z),|a|+|b| \neq 0$ are rational functions. An example is given and a question posed in Section 4.

For the case $n=1$, Wittich [14] treated entire solutions of the functional equation

$$
f(s z)=P_{1}(z) f(z)+P_{0}(z)
$$

Received November 4, 1997, revised May 23, 1998.

1991 Mathematics Subject Classification: 39B10, 30D35.

Key words and phrases: functional equation, meromorphic function, growth of meromorphic function. 
where $P_{1}(z)$ and $P_{0}(z)$ are polynomials and $|s|>1$. Wittich proved that all solutions $f(z)$ of $(1.3)$ satisfy

$$
\log M(r, f) \sim \frac{m}{2 \log |s|}(\log r)^{2}
$$

as $r \rightarrow \infty$ where $m$ is the degree of $P_{1}$.

Theorem 1.1. All meromorphic solutions of $(1.1)$ satisfy $T(r, f)=O\left((\log r)^{2}\right)$.

Theorem 1.2. All transcendental meromorphic solutions of (1.1) satisfy

$$
(\log r)^{2}=O(T(r, f)) .
$$

The main idea in the proofs of Theorems 1.1 and 1.2 is to compare the Nevanlinna functions of $f(z)$ and $f(c z)$. From the definitions, we have $M(r, f(c z))=$ $\max _{|z|=r}|f(c z)|=\max _{|z|=|c| r}|f(z)|=M(|c| r, f)$ and

$$
\begin{aligned}
m(r, f(c z)) & =\frac{1}{2 \pi} \int_{0}^{2 \pi} \log ^{+}\left|f\left(r e^{i \theta}\right)\right| d \theta=\frac{1}{2 \pi} \int_{0}^{2 \pi} \log ^{+}\left|f\left(|c| r e^{i(\theta+\alpha)}\right)\right| d \theta \\
& =m(|c| r, f), \quad \arg c=\alpha .
\end{aligned}
$$

Since the number of poles of $f(z)$ in $\{|z|<|c| r\}$ is equal to the the number of poles of $f(c z)$ in $\{|z|<r\}$, we have $n(|c| r, f)=n(r, f(c z))$, and in particular, $n(0, f)=$ $n(0, f(c z))$. This implies that

$$
\begin{aligned}
N(|c| r, f) & =\int_{0}^{|c| r} \frac{n(t, f)-n(0, f)}{t} d t+n(0, f) \log |c| r \\
& =\int_{0}^{r} \frac{n(s, f(c z))-n(0, f(c z))}{s} d s+n(0, f(c z))(\log r+\log |c|) \\
& =N(r, f(c z))+n(0, f(c z)) \log |c| .
\end{aligned}
$$

Hence, we have $T(r, f(c z))=T(|c| r, f)+O(1)$.

This estimate can be used to show that if all coefficients of (1.1) are constant, then (1.1) has no transcendental meromorphic solution. To do this, we assume that (1.1) with constant coefficients possesses a meromorphic solution $f(z)$. From (1.1), we have

$$
T(r, f) \leq \sum_{j=1}^{n} T\left(r, f\left(c^{j} z\right)\right)+O(1) \leq \sum_{j=1}^{n} T\left(|c|^{j} r, f\right)+O(1) \leq n T(|c| r, f)+O(1)
$$

hence, there exist $B>0$ and $R$ large enough such that

$$
\log T(r, f) \leq \log T(|c| r, f)+B, \quad r \geq R
$$

For any $r \geq R$, there exists an integer $m$ such that

From (1.4) and (1.5),

$$
\frac{R}{|c|^{m-1}} \leq r<\frac{R}{|c|^{m}}
$$

$$
\begin{aligned}
\log T(r, f) & \leq \log T\left(|c|^{m} r, f\right)+m B \\
& \leq \log T(R, f)+B\left(\frac{\log r}{\log \frac{1}{|c|}}-\frac{\log R}{\log \frac{1}{|c|}}+1\right) .
\end{aligned}
$$

It follows that $\log T(r, f)=\left(B / \log \frac{1}{|c|}\right) \log r+O(1)$. This implies that $f(z)$ is a rational function. 


\section{Proofs of Theorem 1.1 and Theorem 1.2}

Proof of Theorem 1.1. Let $s=1 / c$. Choose $R$ so large that $Q$ and the $a_{j}$ have no zeros or poles in $B_{R}:=\{z \in \mathbb{C}:|z| \geq R\}$. If $w \in B_{R}$ and if $s^{j} w$ is not a pole of $f$ for $j=0,1, \ldots, n-1$, then, by (1.1), $s^{n} w$ is not a pole of $f$. It follows by induction that $s^{j} w$ is not a pole of $f$ for any $j \in \mathbb{N}$. Let $w_{1}, \ldots, w_{m}$ be the poles of $f$ in $\{z \in \mathbb{C}: R \leq$ $\left.|z| \leq|s|^{n} R\right\}$. Then all poles of $f$ in $B_{R}$ are contained in $\left\{s^{j} w_{l}: j \in \mathbb{N}, l \in\{1, \ldots, m\}\right\}$. From this, it is not difficult to deduce that $n(r, f)=O(\log r)$. This implies that

$$
N(r, f)=O\left((\log r)^{2}\right) \text {. }
$$

To estimate $m(r, f)$, note that it follows from (1.1) that there exists an $A>0$ such that if $r>s^{n} R$, then

$$
M(r, f) \leq r^{A}\left(\sum_{j=1}^{n} M\left(|c|^{j} r, f\right)+1\right) .
$$

This inequality is useful only if the right-hand side is finite; that is, if $f$ has no poles with modulus $|c|^{j} r, r=1, \ldots, n$. Thus we fix $T \in\left[R,|s|^{n} R\right]$ such that $\left|s^{j} w_{l}\right| \neq T$ for all $j \in\{1, \ldots, n\}$ and $l \in\{1, \ldots, m\}$. Then there is no pole of $f$ with modulus $|s|^{j} T$ for any $j \in \mathbb{N}$. For $k \in \mathbb{N}$, we define

$$
M_{k}=\max _{j=0,1, \ldots, k} M\left(|s|^{j} T, f\right)+1 .
$$

It follows that

$$
\begin{aligned}
M\left(|s|^{k} T, f\right) & \leq\left(|s|^{k} T\right)^{A}\left(\sum_{j=k-n}^{k-1} M\left(|s|^{j} T, f\right)+1\right) \\
& \leq|s|^{k A} T^{A}\left(\sum_{j=k-n}^{k-1} M_{j}+1\right) \\
& \leq|s|^{k A} T^{A}(n+1) M_{k-1}
\end{aligned}
$$

for $k \geq n$ and thus

$$
M_{k} \leq|s|^{B k} M_{k-1}
$$

for some $B>A$ and all $k \in \mathbb{N}$. With $L_{k}=\log M_{k}$ and $C=B \log |s|$, we deduce that

$$
L_{k} \leq C k+L_{k-1} \text {. }
$$

Induction shows that

$$
L_{k} \leq C \sum_{j=1}^{k} j+L_{0}=C \frac{k(k+1)}{2}+L_{0} \leq C k^{2}
$$

for large $k$. It follows that

$$
m\left(|s|^{k} T, f\right) \leq \log M\left(|s|^{k} T, f\right) \leq C k^{2}=C\left(\frac{\log \left(|s|^{k} T\right)-\log T}{\log |s|}\right)^{2} .
$$

Combining (2.1) and (2.2), we obtain

$$
T(r, f)=m(r, f)+N(r, f)=O\left((\log r)^{2}\right)
$$

for $r=|s|^{k} T, k \in \mathbb{N}, k \rightarrow \infty$. Since $T(r, f)$ is increasing, it is now not difficult to see that the last equation also holds if $r \rightarrow \infty$ through any sequence of $r$-values. 
Proof of Theorem 1.2. As before, we put $s=1 / c$ and we choose a large $R$ as in the proof of Theorem 1.1. The arguments used there show that if $f$ has infinitely many poles, then the annulus $\left\{z \in \mathbb{C}: S \leq|z| \leq|s|^{n} S\right\}$ contains a pole of $f$ for all $S \geq R$. We deduce that $\log r=O(n(r, f))$ and this implies that $(\log r)^{2}=O(N(r, f))$ and hence $(\log r)^{2}=O(T(r, f))$.

Hence, we only need to consider the case that $f$ has only finitely many poles and thus may assume without loss of generality that $f$ is entire. We assume that $\left|a_{j}(z)\right| \sim c_{j}|z|^{d_{j}}$ and $|Q(z)| \sim p|z|^{q}$ as $|z| \rightarrow \infty$ where $c_{j}, p>0$ and $d_{j}, q \in \mathbb{Z}, j=0,1, \ldots, n$. Let $d=\max \left\{d_{j}: j=0,1, \ldots, n\right\}$ and $\ell=\min \left\{j: d_{\ell}=d\right\}$. For $i \in \mathbb{N}$, we define

$$
T_{i}=M\left(|s|^{i} R, f\right) \text {. }
$$

Clearly, the $T_{i}$ form an increasing sequence. Since $\log M(r, f)$ is convex in $\log r$ and since $f$ is transcendental,

$$
\frac{M(\alpha r, f)}{M(r, f)} \rightarrow \infty \text { and } \frac{M(r, f)}{r^{\beta}} \rightarrow \infty
$$

as $r \rightarrow \infty$ for each fixed $\alpha, \beta \in \mathbb{R}, \alpha>1$. This implies that

$$
\frac{T_{i}}{T_{i-1}} \rightarrow \infty \text { and } \frac{T_{i}}{\gamma^{i}} \rightarrow \infty
$$

for each $\gamma>0$ as $i \rightarrow \infty$. We write (1.1) in the form

$$
a_{\ell}(z) f\left(c^{\ell} z\right)=-\sum_{j=0}^{\ell-1} a_{j}(z) f\left(c^{j} z\right)-\sum_{j=\ell+1}^{n} a_{j}(z) f\left(c^{j} z\right)+Q(z) .
$$

For $m \in \mathbb{N}, m>\ell$, we choose $z$ such that $|z|=|s|^{m} R$ and $T_{m-\ell}=\left|f\left(c^{\ell} z\right)\right|$ and obtain

$$
\begin{aligned}
& c_{\ell} R^{d}|s|^{m d} T_{m-\ell} \\
& \quad \leq(1+o(1))\left(\sum_{j=0}^{\ell-1} c_{j} R^{d_{j}}|s|^{m d_{j}} T_{m-j}+\sum_{j=\ell+1}^{n} c_{j} R^{d_{j}}|s|^{m d_{j}} T_{m-j}+p R^{q}|s|^{m q}\right)
\end{aligned}
$$

as $m \rightarrow \infty$. Here the first sum is empty if $\ell=0$ and the second one is empty if $\ell=n$. Using (2.3) and the fact that $d_{j} \leq d$ for all $j$, we immediately obtain a contradiction if $\ell=0$. We may thus assume that $\ell>0$ and consider the above estimate for $m=k \ell$ where $k \in \mathbb{N}, k \geq 2$. Using $d_{j} \leq d-1$ for $0 \leq j \leq \ell-1$ and $d_{j} \leq d$ for $\ell+1 \leq j \leq n$, as well as the monotonicity of the $T_{i}$, we obtain

$$
\begin{aligned}
& c_{\ell} R^{d}|s|^{k \ell d} T_{(k-1) \ell} \\
& \quad \leq(1+o(1))\left(\sum_{j=0}^{\ell-1} c_{j} R^{d-1}|s|^{k \ell(d-1)} T_{k \ell}+\sum_{j=\ell+1}^{n} c_{j} R^{d}|s|^{k \ell d} T_{(k-1) \ell-1}+p R^{q}|s|^{k \ell q}\right) .
\end{aligned}
$$

It follows that

$$
T_{(k-1) \ell} \leq A_{1}|s|^{-k \ell} T_{k \ell}+A_{2} T_{(k-1) \ell-1}+A_{3}|s|^{k \ell(q-d)}
$$

with positive constants $A_{j}$. Now the first part of (2.3) implies that

$$
A_{2} T_{(k-1) \ell-1} \leq \frac{1}{3} T_{(k-1) \ell}
$$


and the second part of (2.3) implies that

$$
A_{3}|s|^{k \ell(q-d)}=A_{3}|s|^{\ell(q-d)}|s|^{(k-1) \ell(q-d)} \leq \frac{1}{3} T_{(k-1) \ell}
$$

provided $k$ is large enough. It follows for $k$ sufficiently large that

$$
T_{(k-1) \ell} \leq 3 A_{1}|s|^{-k \ell} T_{k \ell}
$$

We put $S_{k}=T_{k \ell}$ and deduce that if $B<\ell$, then

$$
S_{k} \geq|s|^{B k} S_{k-1}
$$

for all large $k \in \mathbb{N}$. An induction argument similar to the one used in the proof of Theorem 1.1 for $M_{k}$ now implies that $\log S_{k}>C k^{2}$ for some $C>0$ and large $k \in \mathbb{N}$. As before, we can conclude from this first that

$$
\log M(r, f) \geq\left(\frac{C}{(\ell \log |s|)^{2}}-o(1)\right)(\log r)^{2}
$$

for $r=|s|^{k \ell} R, k \in \mathbb{N}, k \rightarrow \infty$, and then deduce again that this holds as $r \rightarrow \infty$ through any sequence of $r$-values. Using the inequality $\log M(r, f) \leq 3 T(2 r, f)$, we see that the last inequality also holds with $\log M(r, f)$ replaced by $T(r, f)$.

\section{Existence of meromorphic solutions}

In this section, we are concerned with an existence theorem for the functional equation (1.2) when $a(z)=\sum_{k=0}^{A} a_{k} z^{k}$ and $b(z)=\sum_{\ell=0}^{B} b_{\ell} z^{\ell}$ are polynomials. We prove

Theorem 3.1. (i) If there exists no integer $p$ satisfying $c^{2 p}+a_{0} c^{p}+b_{0}=0$, then (1.2) does not possess any transcendental meromorphic solution.

(ii) If $b_{0} \neq 0$ and there exists an integer $p$ such that $c^{2 p}+a_{0} c^{p}+b_{0}=0$, then (1.2) possesses a transcendental meromorphic solution.

(iii) If $b_{0}=0$, then (1.2) does not possess any transcendental meromorphic solution.

Proof. We consider a formal solution of (1.2) which is given by a series at the origin. Let $p$ be an integer (negative may be possible). Set

$$
f(z)=\sum_{n=p}^{\infty} \alpha_{n} z^{n}, \quad \alpha_{p} \neq 0
$$

We may avoid the case where $a(z)$ and $b(z)$ are constants, namely we assume that $M:=\max (A, B)>0$ because (1.2) does not possess a transcendental meromorphic solution when $a(z)$ and $b(z)$ are constants. From (1.2),

$$
\sum_{n=p}^{\infty} \alpha_{n} c^{2 n} z^{n}+\left(\sum_{k=0}^{A} a_{k} z^{k}\right)\left(\sum_{n=p}^{\infty} \alpha_{n} c^{n} z^{n}\right)+\left(\sum_{\ell=0}^{B} b_{\ell} z^{\ell}\right)\left(\sum_{n=p}^{\infty} \alpha_{n} z^{n}\right)=0,
$$

that is,

$$
\sum_{n=p}^{\infty} \alpha_{n} c^{2 n} z^{n}+\sum_{n=0}^{\infty}\left(\sum_{k=p}^{\min (A, n-p)} a_{k} \alpha_{n-k} c^{n-k}\right) z^{n}+\sum_{n=0}^{\infty}\left(\sum_{\ell=p}^{\min (B, n-p)} b_{\ell} \alpha_{n-\ell}\right) z^{n}=0
$$

Comparing the coefficients of $z^{n}$, we get for $n=p, p+1, \ldots$,

$$
\alpha_{n} c^{2 n}+\sum_{k=0}^{\min (A, n-p)} a_{k} \alpha_{n-k} c^{n-k}+\sum_{\ell=0}^{\min (B, n-p)} b_{\ell} \alpha_{n-\ell}=0
$$


Putting $n=p$ in (3.2), we have $\alpha_{p}\left(c^{2 p}+a_{0} c^{p}+b_{0}\right)=0$. This implies that $\left|a_{0}\right|+\left|b_{0}\right| \neq 0$ and

$$
c^{2 p}+a_{0} c^{p}+b_{0}=0
$$

Hence, in the case where there is no integer satisfying (3.3), we conclude that (1.2) has no meromorphic solution, which proves (i).

We remark that if (1.1) possesses a local solution, then the global solution can be obtained by meromorphic continuation using equation (1.1). To say this in more detail, we denote the radius of convergence of the local solution at $z=0$ by $R>0$, and define $D_{m}=\left\{|z|<R /|c|^{m}\right\}$, noting that $\bigcup_{m=0}^{\infty} D_{m}=\mathbb{C}$. First, we show that the local solution which exists in $D_{0}$ can be extended into $D_{1}$ by meromorphic continuation. In fact, we define $f(z)$ in $D_{1} \backslash D_{0}$ as follows:

$$
f(z)=-\sum_{j=1}^{n} \frac{a_{j}(z)}{a_{0}(z)} f\left(c^{j} z\right), \quad z \in D_{1} \backslash D_{0} .
$$

The rational functions $a_{j}(z) / a_{0}(z), j=1, \ldots, n$, are, of course, meromorphic in $D_{1}$, and the functions $f\left(c^{j} z\right)$ are also meromorphic in $D_{1}$ since $c^{j} z \in D_{0}, j=1, \ldots, n$. Hence, the right-hand side is defined in $D_{1}$. Therefore, $f(z)$ is meromorphic in $D_{1}$ and satisfies (1.1). Repeating this process we construct a global solution.

Next we prove (ii). We note that if there are two integers $p_{1}, p_{2}$ satisfying (3.3), then we choose $\max \left(p_{1}, p_{2}\right)$ as a $p$ in (3.1). If we choose $\alpha_{p}$ first, then $\alpha_{p+1}, \alpha_{p+2}, \ldots$, are determined by (3.2) recursively. In particular, for the case $n>M$, from (3.2)

$$
\alpha_{n}\left(c^{2 n}+a_{0} c^{n}+b_{0}\right)+\alpha_{n-1}\left(a_{1} c^{n-1}+b_{1}\right)+\cdots+\alpha_{n-M}\left(a_{M} c^{n-M}+b_{M}\right)=0
$$

where $a_{j}=0, j=A+1, \ldots, M$, if $M>A$ and $b_{i}=0, i=B+1, \ldots, M$, if $M>B$. Thus $\alpha_{n}$ depends only on $\alpha_{n-M}, \alpha_{n-M+1}, \ldots, \alpha_{n-1}$. We already have noted that if (1.2) possesses a local solution, in other words, the formal solution (3.1) has a positive convergence radius, then the global solution could be obtained by analytic continuation using equation (1.2). Suppose that (3.1) has no positive convergence radius, say $\lim \sup _{k \rightarrow \infty} \sqrt[k]{\left|\alpha_{k}\right|}=\infty$. Put $\xi_{k}=\max _{p \leq \ell \leq k} \sqrt[\ell]{\left|\alpha_{\ell}\right|}$. Then $\xi_{k} \rightarrow \infty$ as $k \rightarrow \infty$. Choose a subsequence $\left\{\alpha_{k_{j}}\right\}$ such that $\sqrt[k_{j}]{\left|\alpha_{k_{j}}\right|}=\xi_{k_{j}}$ and $\sqrt[k_{j}-i]{\left|\alpha_{k_{j}-i}\right|}<\xi_{k_{j}}$, $i=1, \ldots, M$. Since $\xi_{k_{j}} \rightarrow \infty$ as $j \rightarrow \infty$, from (3.4) we get

$$
\begin{aligned}
& \xi_{k_{j}}^{k_{j}}\left(\left|b_{0}\right|-|c|^{2 k_{j}}-\left|a_{0}\right||c|^{k_{j}}\right) \\
& \quad \leq \xi_{k_{j}}^{k_{j}-1}\left(\left|a_{1}\right||c|^{k_{j}-1}+\left|b_{1}\right|\right)+\cdots+\xi_{k_{j}}^{k_{j}-M}\left(\left|a_{M}\right||c|^{k_{j}-M}+\left|b_{M}\right|\right),
\end{aligned}
$$

hence

$$
\xi_{k_{j}}\left(\left|b_{0}\right|-o(1)\right) \leq\left|b_{1}\right|+\cdots+\left|b_{M}\right|+o(1) .
$$

Since $b_{0} \neq 0$, the inequality above yields a contradiction.

Before we prove (iii), we shall outline the proof here. Assume that there exists a transcendental meromorphic solution $f(z)$. Write $f(z)$ as (3.1) in a neighborhood of the origin. We try to find a contradiction by showing that there exists an integer $N$ such that $\alpha_{n}=0, n \geq N$. To do this, we divide the proof into two steps. First, we show that for any $\eta>0$, there exists a $T>0$ such that

$$
\left|\delta_{n}\right| \leq(1+\eta)^{n} T, \quad \text { for any } n,
$$


where $\delta_{n}=t^{-n} c^{\gamma n^{2}} \alpha_{n}, t$ and $\gamma>0$ are constant. This will be inequality (3.6) in the proof. The second step is that by using the inequality above and (3.4), we show that for any $\nu$

$$
\left|\delta_{n}\right| \leq c_{1}^{n} T T_{1}\left(|c|^{2 \gamma n} T_{1}\right)^{\nu}
$$

where $n$ is fixed and arbitrary, and $c_{1}, T$, and $T_{1}$ are constants independent of $n$ and $\nu$, which will be numbered (3.20) in the proof. Then we can find $N$ such that $\delta_{n}=\alpha_{n}=0$, when $\left.|| c\right|^{2 \gamma n} T_{1} \mid<1, n \geq N$.

Now we start the proof of (iii). Similarly as before, we see that for large $n$, (3.4) holds. Define $K$ to be the smallest integer such that $b_{K}$ does not vanish, i.e., $b_{0}=\cdots=b_{K-1}=0, b_{K} \neq 0$, and set $\gamma=1 /(2 K)$. Note that $a_{0} \neq 0$ in this case. In (3.4), if we put $\alpha_{m}=\beta_{m} c^{-\gamma m^{2}}$ for any $m$, then

$$
\begin{aligned}
& \beta_{n} c^{-\gamma n^{2}}\left(c^{2 n}+c^{n} a_{0}\right)+\cdots+\beta_{n-K+1} c^{-\gamma(n-K+1)^{2}}\left(c^{n-K+1} a_{K-1}\right) \\
& \quad+\beta_{n-K} c^{-\gamma(n-K)^{2}}\left(c^{n-K} a_{K}+b_{K}\right)+\cdots+\beta_{n-M} c^{-\gamma(n-M)^{2}}\left(c^{n-M} a_{M}+b_{M}\right)=0 .
\end{aligned}
$$

Dividing both sides by $c^{-\gamma(n-K)^{2}}$ and arranging as

$$
\begin{aligned}
& \beta_{n} a_{0} c^{K / 2}+\beta_{n-K} b_{K}+\beta_{n} c^{n+K / 2}=-c^{2 \gamma n}\left(\beta_{n-1} a_{1} c^{p_{1}}+\cdots+\beta_{n-K} a_{K} c^{p_{K}}\right. \\
& \left.+\beta_{n-K-1}\left(a_{K+1} c^{n-K-1}+b_{K+1}\right) c^{q_{K+1}}+\cdots+\beta_{n-M}\left(a_{M} c^{n-M}+b_{M}\right) c^{q_{M}}\right)
\end{aligned}
$$

where

$$
\begin{aligned}
& p_{j}=2 n \gamma(j-1)+\gamma K^{2}-\gamma j^{2}-j, \quad \text { for } j=1,2, \ldots, K, \text { and } \\
& q_{j}=n(2 \gamma j-2 \gamma-1)+\gamma\left(K^{2}-j^{2}\right) \text { for } j=K+1, K+2, \ldots, M .
\end{aligned}
$$

Further, we put $-b_{K} /\left(a_{0} c^{K / 2}\right)=t^{K}$ and $\beta_{m}=t^{m} \delta_{m}$ for all $m$. Then we get

$$
\delta_{n}-\delta_{n-K}+\frac{c^{n}}{a_{0}} \delta_{n}=-c^{2 \gamma n}\left(r_{1} \delta_{n-1}+\cdots+r_{M} \delta_{n-M}\right)
$$

where

$$
\begin{aligned}
& r_{j}=\frac{a_{j}}{a_{0} t^{j}} c^{p_{j}-K / 2}, \quad j=1,2, \ldots, K, \\
& r_{j}=\frac{\left(a_{j} c^{n-j}+b_{j}\right)}{a_{0} t^{j}} c^{q_{j}-K / 2}, \quad j=K+1, K+2, \ldots, M .
\end{aligned}
$$

We see that $\left|r_{j}\right| \leq R_{j}$ for some constants $R_{j}$, and note that $R_{j}$ are independent of $n$. In fact, we have

$$
\left|r_{j}\right|=\left|\frac{a_{j}}{a_{0} t^{j}} c^{2 n \gamma(j-1)+\gamma K^{2}-\gamma j^{2}-j-K / 2}\right| \leq \frac{\left|a_{j}\right|}{\left|a_{0}\right||t|^{j}}|c|^{\gamma K^{2}-\gamma j^{2}-j-K / 2},
$$

for $j=1,2, \ldots, K$, and

$$
\left|r_{j}\right|=\left|\frac{\left(a_{j} c^{n-j}+b_{j}\right)}{a_{0} t^{j}} c^{n(2 \gamma j-2 \gamma-1)+\gamma\left(K^{2}-j^{2}\right)-K / 2}\right| \leq \frac{\left(\left|a_{j}\right|+\left|b_{j}\right|\right)}{\left|a_{0}\right||t|^{j}}|c|^{\gamma\left(K^{2}-j^{2}\right)-K / 2},
$$

for $j=K+1, K+2, \ldots, M$. For the sake of brevity, we put $R_{1}+\cdots+R_{M}=L$. We first assert that for any $\eta>0$, there exists $T>0$ such that for any $n$

$$
\left|\delta_{n}\right| \leq(1+\eta)^{n} T .
$$


In fact, for any fixed $\eta$, we find an $n_{0}$ such that for any $m>n_{0}$

$$
\frac{1+|c|^{2 \gamma m} L}{1-|c|^{m} /\left|a_{0}\right|}<1+\eta
$$

We choose $T>0$ large enough such that, for $m \leq n_{0}$,

$$
\frac{\left|\delta_{m}\right|}{(1+\eta)^{m}} \leq T
$$

i.e., (3.6) holds for $m \leq n_{0}$. We suppose that (3.6) holds for all $m \leq n-1$ where $n-1 \geq n_{0}$. Then, from (3.5) and (3.8),

$$
\begin{aligned}
\left|\delta_{n}\right|\left(1-\frac{|c|^{n}}{\left|a_{0}\right|}\right) & \leq\left|\delta_{n}\left(1+\frac{c^{n}}{a_{0}}\right)\right| \leq\left|\delta_{n-K}\right|+c^{2 \gamma n}\left|r_{1} \delta_{n-1}+\cdots+r_{M} \delta_{n-M}\right| \\
& \leq\left|\delta_{n-K}\right|+|c|^{2 \gamma n}(1+\eta)^{n-1} T L \leq(1+\eta)^{n-1} T\left(1+|c|^{2 \gamma n} L\right) .
\end{aligned}
$$

Thus, by (3.7),

$$
\left|\delta_{n}\right| \leq(1+\eta)^{n-1} T\left(\frac{1+|c|^{2 \gamma n} L}{1-|c|^{n} /\left|a_{0}\right|}\right)<(1+\eta)^{n} T .
$$

This implies that (3.6) holds for all $n$, hence the assertion follows. We write the right-hand side of (3.5) simply as $S_{n}$, so that

$$
\delta_{n}-\delta_{n-K}=-\frac{c^{n}}{a_{0}} \delta_{n}+S_{n}
$$

and

$$
\left|S_{n}\right| \leq|c|^{2 \gamma n}(1+\eta)^{n} T L
$$

Using (3.9), we get

$$
-\delta_{n-K}+\delta_{n+m K}=-\frac{1}{a_{0}} \sum_{j=0}^{m} c^{n+j K} \delta_{n+j K}+\sum_{j=0}^{m} S_{n+j K} .
$$

We assert that $\lim _{n \rightarrow \infty} \delta_{n}=0$. In fact, if we assume the contrary, then there exists a subsequence $\left\{n_{k}\right\}$ such that $\left|\delta_{n_{k}}\right|>\varepsilon>0$. Recalling the definition of $\delta_{n}$, namely $\delta_{n}=c^{\gamma n^{2}} \alpha_{n} / t^{n}$, we have

$$
\sqrt[n_{k}]{\left|\alpha_{n_{k}}\right|}=\sqrt[n_{k}]{\left|\frac{t^{n_{k}}}{c^{\gamma n_{k}^{2}}} \delta_{n_{k}}\right|} \geq \frac{|t|}{|c|^{\gamma n_{k}}} \sqrt[n_{k}]{\varepsilon} \rightarrow \infty(\text { as } k \rightarrow \infty) .
$$

This gives $1 / \lim \sup _{n \rightarrow \infty} \sqrt[n]{\left|\alpha_{n}\right|} \leq \lim _{k \rightarrow \infty} 1 / \sqrt[n_{k}]{\mid \alpha_{n_{k} \mid}}=0$, which implies that the convergence radius is zero, a contradiction. Hence, letting $m \rightarrow \infty$ in (3.11), we get

$$
-\delta_{n-K}=-\frac{1}{a_{0}} \sum_{j=0}^{\infty} c^{n+j K} \delta_{n+j K}+\sum_{j=0}^{\infty} S_{n+j K} .
$$

For simplicity, we denote by $\Sigma_{1}$ and $\Sigma_{2}$ the first and the second sum in the right-hand side of (3.12), respectively. From (3.6) and (3.10),

$$
\begin{aligned}
& \left|\Sigma_{1}\right| \leq \frac{1}{\left|a_{0}\right|} \sum_{j=0}^{\infty}|c|^{n+j K}\left|\delta_{n+j K}\right| \leq \frac{(|c|(1+\eta))^{n}}{\left|a_{0}\right|} T \sum_{j=0}^{\infty}(|c|(1+\eta))^{j K}, \\
& \left|\Sigma_{2}\right| \leq \sum_{j=0}^{\infty}\left|S_{n+j K}\right| \leq\left(|c|^{2 \gamma}(1+\eta)\right)^{n} T L \sum_{j=0}^{\infty}\left(|c|^{2 \gamma}(1+\eta)\right)^{j K} .
\end{aligned}
$$


Put $c_{1}:=|c|^{2 \gamma}(1+\eta)>|c|(1+\eta)$. Then, for a sufficiently small $\eta>0$, we may assume that $c_{1}<1$. From (3.11), (3.13), and (3.14),

$$
\left|\delta_{n-K}\right| \leq\left|\Sigma_{1}\right|+\left|\Sigma_{2}\right| \leq \frac{c_{1}^{n}}{\left|a_{0}\right|} T \sum_{j=0}^{\infty} c_{1}^{K j}+c_{1}^{n} T L \sum_{j=0}^{\infty} c_{1}^{K j}=c_{1}^{n} T\left(\frac{1}{\left|a_{0}\right|}+L\right)\left(\frac{1}{1-c_{1}^{K}}\right)
$$

Set $\left(\frac{1}{\left|a_{0}\right|}+\frac{L}{c_{1}^{M}}\right)\left(\frac{1}{1-c_{1}^{K}}\right)=T_{1}$. Then we have $\left|\delta_{n-K}\right| \leq c_{1}^{n} T T_{1}$. Thus, we see that

$$
\left|\delta_{n}\right| \leq c_{1}^{n} T T_{1}
$$

Using (3.15) instead of (3.6), we estimate $S_{n}, \Sigma_{1}$ :

$$
\begin{gathered}
\left|S_{n}\right| \leq|c|^{2 \gamma n} c_{1}^{n-M} T T_{1} L \\
\left|\Sigma_{1}\right| \leq \frac{1}{\left|a_{0}\right|} \sum_{j=0}^{\infty}|c|^{n+j K} c_{1}^{n+j K} T T_{1} \leq \frac{|c|^{n} c_{1}^{n}}{\left|a_{0}\right|} T T_{1} \sum_{j=0}^{\infty}\left(|c| c_{1}\right)^{j K} .
\end{gathered}
$$

From (3.16),

$$
\left|\Sigma_{2}\right| \leq \sum_{j=0}^{\infty}\left|S_{n+j K}\right| \leq|c|^{2 \gamma n} c_{1}^{n-M} T T_{1} L \sum_{j=0}^{\infty}\left(|c|^{2 \gamma} c_{1}\right)^{j K}
$$

Combining (3.12), (3.17), and (3.18), we have

$$
\begin{aligned}
\left|\delta_{n-K}\right| & \leq\left|\Sigma_{1}\right|+\left|\Sigma_{2}\right| \leq \frac{|c|^{n} c_{1}^{n}}{\left|a_{0}\right|} T T_{1} \sum_{j=0}^{\infty} c_{1}^{j K}+|c|^{2 \gamma n} c_{1}^{n-M} T T_{1} L \sum_{j=0}^{\infty} c_{1}^{j K} \\
& \leq|c|^{2 \gamma n} c_{1}^{n} T T_{1}\left(\frac{1}{\left|a_{0}\right|}+\frac{L}{c_{1}^{M}}\right)\left(\frac{1}{1-c_{1}^{K}}\right) \leq|c|^{2 \gamma n} c_{1}^{n} T T_{1}^{2} .
\end{aligned}
$$

Thus, we obtain

$$
\left|\delta_{n}\right| \leq|c|^{2 \gamma n} c_{1}^{n} T T_{1}^{2}
$$

Similarly, we get $\left|\delta_{n}\right| \leq|c|^{4 \gamma n} c_{1}^{n} T T_{1}^{3}$ by using (3.19) to estimate $S_{n}, \Sigma_{1}$, and $\Sigma_{2}$. Repeating this process, we obtain for any $\nu \in \mathbb{N}$ :

$$
\left|\delta_{n}\right| \leq|c|^{2 \gamma n \nu} c_{1}^{n} T T_{1}^{\nu+1}=c_{1}^{n} T T_{1}\left(|c|^{2 \gamma n} T_{1}\right)^{\nu}
$$

We now choose $N$ such that $|c|^{2 \gamma n} T_{1}<1$ for $n \geq N$. Letting $\nu \rightarrow \infty$ in (3.20) for $n \geq N$, we see that $\delta_{n}=0$ for $n \geq N$. This implies that $\alpha_{n}=0$ for $n \geq N$, so the solution is a rational function, a contradiction. 


\section{An example and a question}

We consider the specific equation

$$
f\left(c^{2} z\right)-z f(c z)-b f(z)=0, \quad 0<|c|<1
$$

where $b$ is a constant satisfying $c^{p}-b=0$ for some integer $p$. By Theorem 3.1, (4.1) possesses a meromorphic solution $f(z)$. If we write $f(z)$ in the neighborhood of the origin as (3.1), then from (3.3) for any $n>p$, we have

$$
\alpha_{n}\left(c^{2 n}-b\right)-\alpha_{n-1} c^{n-1}=0 .
$$

Hence, we see that $\alpha_{n-1} / \alpha_{n}=\left(c^{2 n}-b\right) / c^{n-1} \rightarrow \infty$, which implies that the convergence radius of (3.1) is $\infty$. Further, from (4.2), we obtain

$$
\alpha_{n}=\frac{\prod_{k=p+1}^{n} c^{k-1}}{\prod_{k=p+1}^{n}\left(c^{2 k}-b\right)} \alpha_{p}=\frac{\frac{1}{(-b)^{n}} \prod_{k=1}^{n} c^{k-1}}{\prod_{k=p+1}^{n}\left(\left(c^{2}\right)^{k}\left(\frac{-1}{b}\right)+1\right)} \frac{(-b)^{p} \alpha_{p}}{\prod_{k=1}^{p} c^{k}} .
$$

Since $\sum_{k=p}^{\infty}\left|c^{2}\right|^{k}<\infty$, we see that $\prod_{k=p+1}^{\infty}\left(\left(c^{2}\right)^{k}\left(\frac{-1}{b}\right)+1\right)$ converges to a finite value. Hence, using $c^{p}=b$, we find that

$$
\left|\alpha_{n}\right| \sim H|c|^{n(n-1) / 2-2 n p}
$$

as $n \rightarrow \infty$, for a positive constant $H$. Let

$$
\mu(r, f)=\max _{n}\left|\alpha_{n} r^{n}\right|
$$

be the maximum term. A computation shows that the maximum of the right-hand side of (4.3) is attained for

$$
n=\frac{\log r}{-\log |c|}+O(1)
$$

and this leads to

$$
\log \mu(r, f) \sim \frac{(\log r)^{2}}{-2 \log |c|}
$$

This implies that

$$
T(r, f) \sim \log M(r, f) \sim \frac{(\log r)^{2}}{-2 \log |c|}
$$

see, e.g., Jank and Volkman [5, Satz 4.6], or Hayman [4, §4].

Wittich [14] proved that transcendental entire solutions of (1.3) are hypertranscendental. Ritt [8] proved that meromorphic solutions of the Schröder equation $f(c z)=R(f(z))$ where $R(z)$ is a rational function in $z$ are hypertranscendental, except for certain cases where they are given in terms of exponential, trigonometric, or elliptic functions. As Rubel posed in [9, 10], there is an open problem on hypertranscendency for the equation $f(\lambda z)=R(z, f(z))$ where $\lambda$ is a complex constant and $R(z, f)$ is a rational function in $z$ and $f$. We mention other articles or expositions for the study of hypertranscendency of solutions of some functional equations, for instance, Becker and Bergweiler [1, 2], Laine [6, Chapter 14], Takano [11], and Yanagihara [12, 13]. Finally we pose a question : What can we say about the hypertranscendency for solutions of $(1.1) ?$ 
Acknowledgment. The authors would like to thank the referee for his kind suggestions. The authors also would like to express our gratitude to Professors Mourad Ismail and Yikman Chiang for drawing our attention to the functional equation, and also thank them for their careful reading of our manuscript.

This work was supported in part by a Grant-in-Aid for General Scientific Research from the Ministry of Education, Science and Culture 08740117 and by a grant from the Nippon Institute of Technology 102 (1996).

\section{References}

1. P.-G. Becker and W. Bergweiler, Hypertranscendency of conjugacies in complex dynamics, Math. Ann. 301 (1995), 463-468.

2. W. Bergweiler, Solution of a problem of Rubel concerning iteration and algebraic differential equations, Indiana Univ. Math. Jour. 44 (1995), 257-267.

3. H. K. Hayman, Meromorphic Functions, Clarendon Press, Oxford, 1964.

4. The local growth of power series: a survey of the Wiman-Valiron method, Can. Math. Bull. 17 (1974), 317-358.

5. G. Jank and L. Volkmann, Meromorphe Funktionen und Differentialgleichungen, BirkhäuserVerlag, Basel-Boston-Stuttgart, 1985.

6. I. Laine, Nevanlinna Theory and Complex Differential Equations, W. de Gruyter, Berlin-New York, 1992.

7. R. Nevanlinna, Analytic Functions, Springer Verlag, Berlin-Heidelberg-New York, 1970.

8. J. F. Ritt, Transcendental transcendency of certain functions of Poincaré, Math. Ann. 95 $(1925 / 26), 671-682$.

9. L. A. Rubel, Some research problems about algebraic differential equations, Trans. Amer. Math. Soc. 280 (1983), 43-52.

10. (1992), 659-680.

11. K. Takano, On the hypertranscendency of solutions of a difference equation of Kimura, Funkcial. Ekvac. 16 (1993), 241-254.

12. N. Yanagihara, Hypertranscendency of solutions of some difference equations, Japan. J. Math. 17 (1981), 109-168.

13. - Meromorphic solutions of some functional equations, Bull. Sc. Math., $2^{\mathrm{e}}$ série 107 (1983), 289-300.

14. H. Wittich, Bemerkung zu einer Funktionalgleichung von H. Poincaré, Arch. Math. 2 (1949/1950), 90-95.

Mathematisches Seminar, Christian-Albrechts-Universität zU Kiel, Ludewig-MeYn-Str. 4, D-24098 KIEL, GERMANY

E-mail: bergweiler@math.uni-kiel.de

Department of Mathematics, Nippon Institute of Technology, 4-1 Gakuendai Miyashiro, Minamisaitama Saitama, 345 JAPAN

E-mail: ishi@nit.ac.jp

Department of Mathematics, Faculty of Science, Chiba University, 1-33 Yayoi-cho Inage, CHIBA 263, JAPAN

E-mail: yanagi@math.s.chiba-u.ac.jp 